\title{
A rare case of acute necrotizing eosinophilic myocarditis: Bridge to recovery with ventricular assist device support
}

\author{
Jaromir Kohout, MD, Francis D. Ferdinand, MD, Shotaro Imaizumi, MD, Elena C. Holmes, NP, \\ and Louis E. Samuels, MD, Wynnewood, Pa
}

A

cute necrotizing eosinophilic myocarditis is a rare disorder characterized by fulminant congestive heart failure, usually in the absence of directly related extracardiac disease. If diagnosed before death, the mainstay of therapy is steroid administration. Often, the disease is fatal and death results from cardiogenic shock. ${ }^{1}$ Management, therefore, consists of circulatory support, confirmed pathologic diagnosis, and steroid administration. The purpose of this report is to describe the role of ventricular assist device support for this rare disorder.

\section{Clinical Summary}

A 37-year-old man came to the emergency department with shortness of breath and cough of 1 week's duration. The medical history was significant for smoking and alcohol abuse. The patient had no known history of illicit drug use or medication allergies. He was recently treated for an upper respiratory tract infection with erythromycin as an outpatient. He returned to the emergency department with chills, fever, headache, ptosis of the left eye, shortness of breath, and chest pain. The patient was admitted and placed on a regimen of intravenous vancomycin, ceftriaxone, and doxycycline. A head computed tomogram and spinal tap were negative for any intracranial disease. After admission, ventricular tachycardia developed, which resulted in cardiac arrest requiring defibrillation, intubation, and cardiopulmonary resuscitation. The electrocardiogram showed marked ST-segment elevation in leads $V_{1}$ to $V_{4}$. Emergent coronary angiography showed no significant coronary artery disease; there was severe left ventricular dysfunction with an ejection fraction of $10 \%$. The patient underwent intra-aortic balloon pump placement and was transferred to Lankenau Hospital, Wynnewood, Pennsylvania, for further management.

Echocardiography showed concentric left ventricular hypertrophy, global hypokinesis, severe left ventricular dysfunction, no significant valvular disease, and no congenital cardiac disease. The right ventricle was mildly dilated and markedly hypokinetic. The admission cardiac index was $1.6 \mathrm{~L} \cdot \mathrm{min}^{-1} \cdot \mathrm{m}^{-2}$ on multiple inotropes and vasopressors. Right heart catheterization demonstrated a right atrial pressure of $30 \mathrm{~mm} \mathrm{Hg}$ and pulmonary artery

From the Lankenau Hospital, Division of Thoracic Surgery, Main Line Health Heart Center, Wynnewood, Pa.

Received for publication April 20, 2006; accepted for publication May 9, 2006.

Address for reprints: Louis E. Samuels, MD, The Lankenau Hospital, Department of Cardiothoracic Surgery, MSB Suite 280, 100 Lancaster Ave, Wynnewood, PA 19096 (E-mail: SamuelsL@mlhs.org).

J Thorac Cardiovasc Surg 2006;132:965-6

$0022-5223 / \$ 32.00$

Copyright (C) 2006 by The American Association for Thoracic Surgery

doi:10.1016/j.jtcvs.2006.05.058

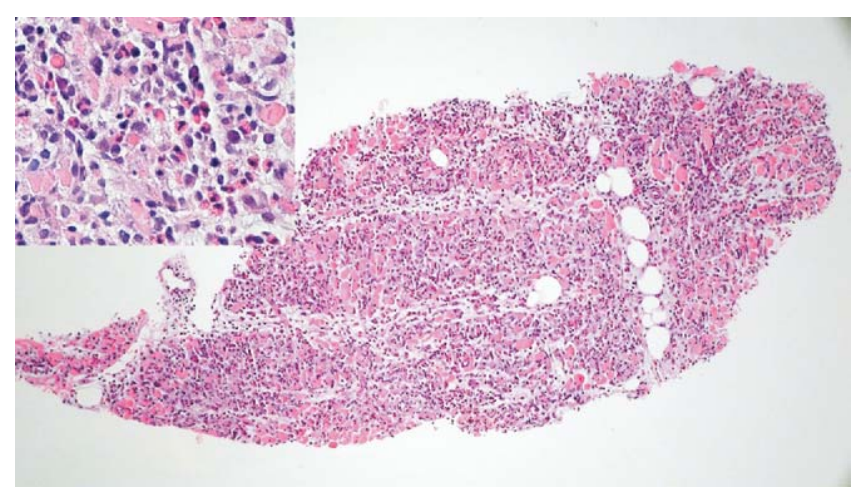

Figure 1. Initial ventricular biopsy showing extensive eosinophilrich inflammatory infiltrate with reactive change associated with edema of the interstitium and dropout of cardiac fibers. Hematoxylin-eosin stain.

systolic pressure of $39 \mathrm{~mm} \mathrm{Hg}$. Troponin levels were elevated at $60.87 \mathrm{ng} / \mathrm{mL}$. Shortly after arrival in the intensive care unit, the patient had another cardiac arrest requiring defibrillation and cardiopulmonary resuscitation. He was taken to the operating room on an emergency basis and placed on cardiopulmonary bypass. A Thoratec left ventricular assist device (Thoratec Corporation, Pleasanton, Calif) was implanted. Myocardial biopsy specimens of both ventricles were obtained. Pathologic evaluation showed extensive eosinophil-rich inflammatory infiltrates associated with interstitial edema as well as dropout of cardiac fibers consistent with acute eosinophilic necrotizing myocarditis (Figure 1). The patient's peripheral eosinophil count was within normal limits. Antibody titers for adenovirus AB, parvovirus, coxsackievirus B1 to B6, Epstein-Barr virus, cytomegalovirus, Mycobacterium, and Lyme disease were negative. The patient was started on a regimen of $250 \mathrm{mg}$ of intravenous methylprednisolone 4 times a day for 3 days and then was continued on a tapering dose for an additional 3 days (60 mg once a day, $40 \mathrm{mg}$ once a day, and $20 \mathrm{mg}$ once a day).

Postoperatively, the patient recovered uneventfully with normal neurologic and end-organ function. The patient continued to receive intravenous steroid therapy. Weekly echocardiograms showed improvement of cardiac function. After 1 month, the echocardiogram showed normalization of cardiac function with minimal ventricular assist device flow. The patient was taken to the operating room for ventricular assist device explantation and repeat myocardial biopsy. Explantation was successful and repeat biopsy showed improvement of the inflammatory component with virtual absence of eosinophils (Figure 2). The patient was discharged and remains active and well at 28 months' follow-up. 


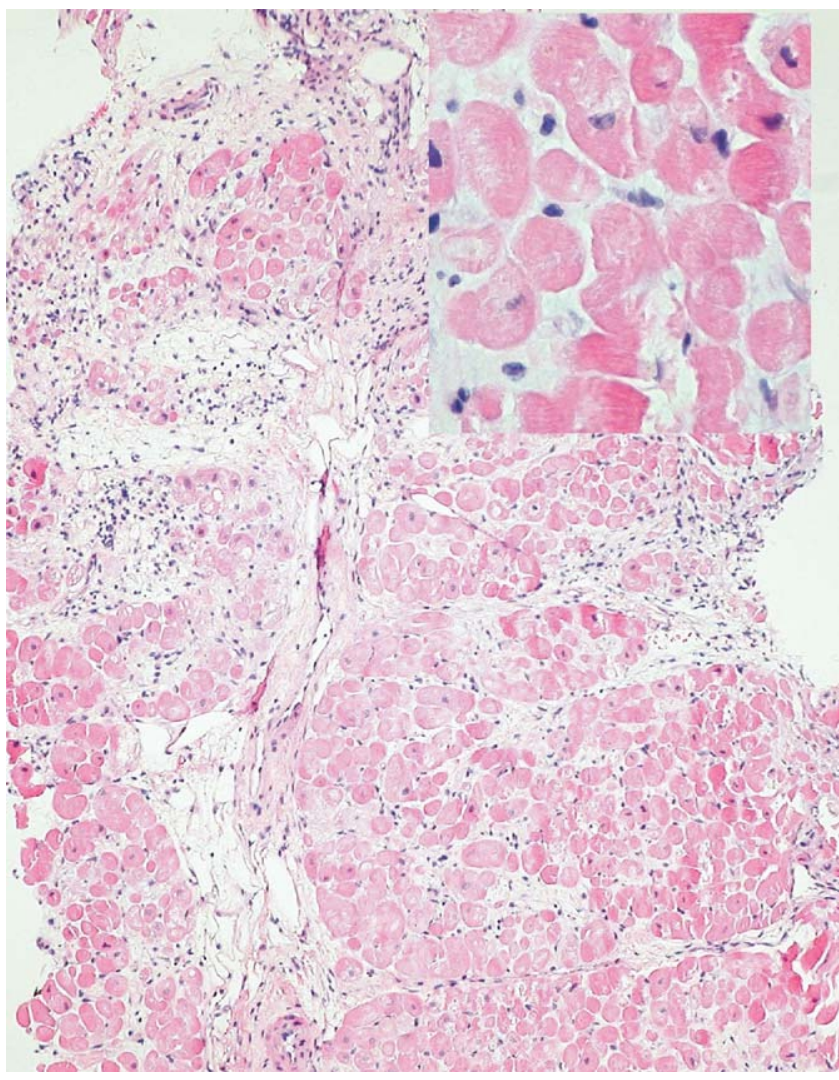

Figure 2. Repeat ventricular biopsy obtained at the time of left ventricular assist device explantation showing dramatic improvement with absence of eosinophils. Hematoxylin-eosin stain.

\section{Comment}

Acute necrotizing eosinophilic myocarditis is a rare disorder of unknown etiology, frequently resulting in cardiogenic shock and fulminant clinical deterioration. Predisposing factors may include viral infection, history of allergic diathesis, or initiation of new medications. ${ }^{1}$ The diagnosis is established only by myocardial biopsy showing the presence of interstitial inflammatory infiltrate composed of eosinophils with associated myocardial necrosis. ${ }^{1}$ Noninvasive cardiac imaging (eg, echocardiography) is necessary to assess severity of cardiac impairment. ${ }^{2}$ The electrocardiographic changes, malignant arrhythmias, and elevated cardiac markers are frequently part of the clinical presentation. Increased wall thickness resulting from inflammation and restricted systolic ventricular function are frequent findings on echocardiography. ${ }^{3}$

The differential diagnosis includes other types of myocarditis, Churg-Strauss syndrome, hypersensitivity reaction, malignant disease, parasitic infection, or hypereosinophilic syndrome. Eosinophilic myocarditis associated with hypereosinophilic syndrome is usually less acute and less severe than acute necrotizing eosinophilic myocarditis. ${ }^{2}$ Association with systemic disease as well as extracardiac involvement is unusual.

Successful treatment of fulminant myocarditis with a biventricular assist device was described for both idiopathic ${ }^{4}$ and acute necrotizing eosinophilic myocarditis. ${ }^{2}$ Heart transplantation can be an option for refractory cases. ${ }^{5}$ Current recommendations for conservative management consist of high-dose steroid administration, diuretics, and inotropic support. Mechanical circulatory support can be a lifesaving bridge in refractory cases until sufficient myocardial recovery occurs.

\section{References}

1. Watanabe N, Nakagawa S, Fukunaga T, Fukuoka S, Hatakeyama K, Hayashi T. Acute necrotizing eosinophilic myocarditis successfully treated by high dose methylprednisolone. Jpn Circ J. 2001;65:923-6.

2. Cooper LT, Zehr KJ. Biventricular assist device placement and immunosuppression as therapy for necrotizing eosinophilic myocarditis. Nat Clin Pract Cardiovasc Med. 2005;2:544-8.

3. Adsett M, West MJ, Galbraith A, Duhig E, Lange A, Palka P. Eosinophilic heart: marked left ventricular wall thickening and myocardial dysfunction improving with corticosteroid therapy. Echocardiography. 2003;20:369-74.

4. Grinda JM, Chevalier P, D'Attellis N, Bricourt MO, Berrebi A, Guibourt $\mathrm{P}$, et al. A fulminant myocarditis in adults and children: biventricular assist device for recovery. Eur J Cardiothorac Surg. 2004; 26:1169-73

5. Touze JE, Debonne JM, Scheiner C, Vaillant A, Sans P, Van de Walle JP, et al. Acute necrotizing eosinophilic myocarditis. Favorable clinical course after heart transplantation. [In French] Presse Med. 1992;21: $565-8$ 